\title{
Conformal radiotherapy plans for palliative bone metastasis - comparison of dosimetric parameters
}

\author{
Gupta N. ${ }^{1}$, Kumar P. ${ }^{2 *}$, Kumar P. ${ }^{3}$, Navitha S. ${ }^{4}$, Mehta A. ${ }^{5}$, Chauhan A. ${ }^{6}$ \\ DOI: https://doi.org/10.17511/ijmrr.2020.i03.02 \\ 1 Naina Gupta, Junior Resident, Department of Radiation Oncology, Shri Ram Murti Smarak Institute of Medical Sciences, Bareilly, Uttar \\ Pradesh, India. \\ 2* Pavan Kumar, Assistant Professor, Department of Radiation Oncology, Shri Ram Murti Smarak Institute of Medical Sciences, Bareilly, \\ Uttar Pradesh, India. \\ 3 Piyush Kumar, Professor and Head, Department of Radiation Oncology, Shri Ram Murti Smarak Institute of Medical Sciences, Bareilly, \\ Uttar Pradesh, India. \\ 4 Navitha S., Assistant Professor Cum Medical Physicist, Department of Radiation Oncology, Shri Ram Murti Smarak Institute of Medical \\ Sciences, Bareilly, Uttar Pradesh, India. \\ 5 Ankita Mehta, Junior Resident, Department of Radiation Oncology, Shri Ram Murti Smarak Institute of Medical Sciences, Bareilly, Uttar \\ Pradesh, India. \\ 6 Arvind Chauhan, Professor, Department of Radiation Oncology, Shri Ram Murti Smarak Institute of Medical Sciences, Bareilly, Uttar \\ Pradesh, India.
}

Background: Palliative radiotherapy offers significant relief in the huge physical distress of patients with bony metastasis. The enormous potential of conformal techniques has not been tested in palliative settings. However,the increasing life span of patients with metastatic disease demands to optimize the radiotherapy techniques to provide maximal durable symptomatic relief. Materials and Methods: Ten patients of vertebral bony metastasis were retrospectively selected and four virtual 3DCRT plans were generated for each patient. The field approaches were a single field, two fields, three fields and five field approaches. Results: A total of forty radiotherapy plans were generated. PTV parameters were significantly better with two field plans over one field plans in terms of $D 90$ ( $p=$ $0.002)$, D50 $(p=0.02)$, Dmean $(p=0.0009)$. Dmeanwassignificantly better with three field approach compared to two field approach $(p=0.0006)$. Conclusion: The three field plans showed better dose distribution to the PTV with an acceptable increase in the dose to OAR's.

Keywords: Conformal radiotherapy plans, Bone metastasis, Dosimetric parameters

Corresponding Author

Pavan Kumar, Assistant Professor, Department of Radiation Oncology, Shri Ram Murti Smarak Institute of Medical Sciences, Bareilly, Uttar Pradesh, India. Email: pawan.mehrotra12@rediffmail.com
How to Cite this Article

Gupta N, Kumar P, Kumar P, Navitha S, Mehta A, Chauhan A. Conformal radiotherapy plans for palliative bone metastasis - comparison of dosimetric parameters. Int J Med Res Rev. 2020;8(3):228-233. Available From

https://ijmrr.medresearch.in/index.php/ijmrr/article/ view/1197
To Browse

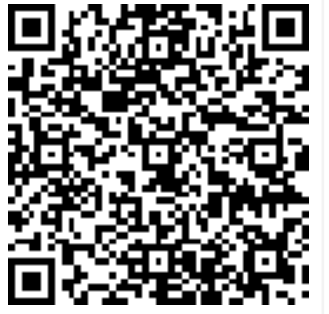

Manuscript Received 2020-05-15

Conflict of Interest No
Review Round 1 2020-05-25

Funding

Nil

Review Round 2
2020-06-01
$\begin{gathered}\text { Ethical Approval } \\ \text { Yes }\end{gathered}$

Review Round 3

Accepted 2020-06-06

Plagiarism $\mathrm{X}$-checker $7 \%$

(c) 2020 by Naina Gupta, Pavan Kumar, Piyush Kumar, Navitha S., Ankita Mehta, Arvind Chauhan and Published by Siddharth Health Research and Social Welfare Society. This is an Open Access article licensed under a Creative Commons Attribution 4.0 International License https://creativecommons.org/licenses/by/4.0/ unported [CC BY 4.0]. 


\section{Background}

Bone metastasis commonly occurs in advanced breast cancer, prostate cancer, and lung cancer [1]. It can lead to huge physical distress owing to the severe intractable pain, symptoms of cord compression with neurological deficits, pathological fractures, and restricted mobility [2].

The quality of life can be adversely impacted due to dependence on routine activities of daily life further leading to psychosocial distress. Palliative radiotherapy provides pain relief to these patients in $50-80 \%$ of patients [3].

The conventional single field approach has been the most commonly utilized technique for palliative radiotherapy of bony metastasis. However, it leads to possible under dosage of the target region and overdosage of the surrounding normal tissues and critical organs.

The newer conformal radiotherapy techniques are being widely practiced in radical settings while they have not been much recognized in palliative settings. Recent trends showed an upsurge in the life span of patients with bony metastasis because of the emergence ofnewer chemotherapy and targeted agents $[4,5]$. The merits of conformal techniques are better dose distribution to the target and better sparing of normal tissues and Organs at risk (OAR's).

This necessitates exploring the advantage of3Dimensional Conformal technique (3DCRT) in palliative settings aiming for increased efficacy and lessenedtoxicity. Increasing the number of fields compared to the usually practiced single beam arrangement may provide better outcomes by improving the coverage of target volume.

However, before clinical adoption, the impact of multibeam arrangement on dosimetry needs to be evaluated. The present study is designed to compare four different 3DCRT plans with different beam arrangements in terms of dose to the target region of interest and organ at risk (OAR's).

\section{Materials and Methods}

Study Setting: Department of Radiation Oncology, Shri Ram Murti Smarak Institute of Medical Sciences

Study Design: A retrospective comparative study.

Study Population: Patients with vertebral bony metastasis treated by Palliative Radiotherapy
Treated between the year 2018 to 2019.

Study tool: Dosimetric parameters were evaluated.

Ten patients of vertebral bony metastasis were retrospectively selected. These patients had undergone simulation in a supine position with arms overhead. Non-Contrast CT scan of thorax or abdomen depending upon the site of vertebral metastasis of $3 \mathrm{~mm}$ slice thickness was obtained.

The clinical target volume (CTV) was delineated which was defined as the involved vertebrae along one vertebra superior and inferior to the involved vertebrae. The Planning target volume (PTV) was made which was a $5 \mathrm{~mm}$ isotropic margin from the CTV. The Organs at risk (OAR's) delineated were bowel, esophagus, combined kidneys, and combined lungs.

The esophagus was delineated cranially from the caudal end of the cricoid cartilage and extending caudally till the gastroesophageal junction. The bowel was delineated as an entirely empty space within the peritoneal cavity extending $1 \mathrm{~cm}$ above and below the PTV.

For each patient, four virtual 3DCRT plans were generated with different types of beam arrangements (total $=40$ plans).

The plans were generated using the following beam arrangements:-

- One field plan- single posteroanterior field (PA Field)at gantry angle 180 degrees (Figure 1 )

- Two field plans- Unequally weighted posteroanterior beam (PA field) and anteroposterior beam (AP field)at gantry angle 180 degrees and 0 degrees respectively. Relative weightage of the posterior beam was 1.3 andthe anterior beam was 0.7 (Figure 2 )

- Three field plan -One anterior and two posterior obliques beams (gantry angles 135 and 225 degrees). Enhanced dynamic wedges of 45 degrees were used with posterior oblique beams. (Figure 3)

- Five field plan -five beams with gantry angles of $45,135,180,225$, and 315 degrees. Enhanced dynamic wedges of 45-degree angles were used with posterior oblique beams. (Figure 4)

The prescribed total dose was $30 \mathrm{~Gy}$ in 10 fractions @3Gy/fraction which was to be delivered in two weeks. Planning was done on the Eclipse treatment planning system 13.6. The calculation algorithm 
Used was AAA. Dose-volume histograms were computed for the various treatment plans and compared for dosimetric analysis.

Data Collection: The following dose-volume parameters were evaluated for the PTV and OAR's

- PTV- D90, D50, Dmean, Conformity index (CI)

Conformity index $=$ Treated volume (absolute volume in CC) (ICRU 62) [6]

PTV volume (absolute volume in $\mathrm{cc}$ )

- Dmeanwas calculated for organs at risk - Bowel, Kidneys, Lungs

Statistical Analysis: The statistical significance was calculated by using the ANOVA test with a pvalue of $<0.05$ considered significant.

Ethical consideration: The study was approved by the Institutional ethical committee prior to its inception.

\section{Results}

Forty virtual radiotherapy plans were generated for ten patients. Out of these ten patients, seven patients had lumbar vertebral metastasis and three patients had thoracic vertebral metastasis.

The dosimetric analysis for PTV is shown in Table 1 and the comparison for statistical significance is done in Table 1 and Table 2.

Table-1: Table depicting parameters showing coverage of PTV (Mean $\mathbf{\pm 2}$ SD).

\begin{tabular}{|l|c|c|c|c|}
\hline & \multicolumn{1}{|c|}{ Field } & \multicolumn{1}{c|}{ 2 Field } & 3 Field & 5 Field \\
\hline D90 & $27.02+1.53$ & $28.95+0.28$ & $29.01+0.44$ & $29.11+0.62$ \\
\hline Dmean & $30.89+1.14$ & $29.10+0.48$ & $30.15+0.19$ & $30.24+0.42$ \\
\hline D50 & $31.03+1.20$ & $30.27+0.45$ & $30.28+0.25$ & $30.36+0.47$ \\
\hline Conformity index & $1.27+0.32$ & $1.97+0.57$ & $1.37+0.18$ & $1.38+0.29$ \\
\hline
\end{tabular}

Table-2: Table depicting statistical analysis of parameters showing coverage of PTV.

\begin{tabular}{|l|l|l|l|}
\hline & $\mathbf{1}$ field vs. $\mathbf{2}$ field & $\mathbf{2}$ field vs. $\mathbf{3}$ field & $\mathbf{3}$ field vs. $\mathbf{5}$ field \\
\hline D90 & 0.002 & 0.78 & 0.62 \\
\hline Dmean & 0.0009 & 0.0006 & 0.64 \\
\hline D50 & 0.02 & 0.98 & 0.66 \\
\hline Conformity index & 0.005 & 0.009 & 0.79 \\
\hline
\end{tabular}

The Dmean for organs at risk for four different radiotherapy plans (esophagus, bowel, lungs, and kidneys) has been shown in Table 3. The differences in mean doses have been compared in various radiotherapy plans for statistical significance in Table 3 and Table 4.
Table-3: Table depicting dose to organs at risk (Mean \pm 2 SD).

\begin{tabular}{|l|c|c|c|c|}
\hline Mean dose of OAR's & \multicolumn{1}{|c|}{ Field } & 2 Field & 3 Field & 5 Field \\
\hline Esophagus & $18.20+3.95$ & $16.47+9.29$ & $18.33+4.46$ & $17.83+4.57$ \\
\hline Lungs & $6.74+1.06$ & $9.81+4.48$ & $9.40+1.29$ & $10.81+1.68$ \\
\hline Bowel & $8.56+2.19$ & $10.15+2.12$ & $10.16+2.53$ & $10.99+3.17$ \\
\hline Kidneys & $2.93+2.25$ & $2.95+2.19$ & $8.72+4.99$ & $9.93+5.22$ \\
\hline
\end{tabular}

Table-4: Table depicting a statistical comparison of OAR's (bowel and kidneys).

\begin{tabular}{|l|l|l|l|}
\hline OAR's & $\mathbf{1}$ field vs. $\mathbf{2}$ field & $\mathbf{2}$ field vs. $\mathbf{3}$ field & $\mathbf{3}$ field vs. $\mathbf{5}$ field \\
\hline Bowel & 0.0002 & 0.984 & 0.027 \\
\hline Kidneys & 0.77 & 0.007 & 0.03 \\
\hline
\end{tabular}

The two field techniques showed a significant improvement in the dose distribution of the PTV indosimetricparameters ofD90, D50, and Dmeanover the one field plan ( $p<0.02$ )(Table 2). This was accompanied by a significant rise in the dose to bowel while the dose to kidneys remained nearly comparable. (Table 4).

The three fieldradiotherapy plans further improved the dose distribution to the PTVwith significantly higher values ( $p=0.0006)$ of mean dose compared to two beam plans with $90 \%$ of the volume receiving more than $95 \%$ of the prescribed dose.The conformity was also seen to be significantly improved $(p=0.009)$ in comparison to the two field plans (Table 2).On the contrary, there wasa significant rise in dose to bowels and kidney.

There was no significant advantage of five field plans over the three field plans in terms of coverage of the PTV (Table 2). Infact, the five field plans had a disadvantage of demonstrating asignificant rise in the dose to kidneys and bowel over the three-field technique (Table 4).

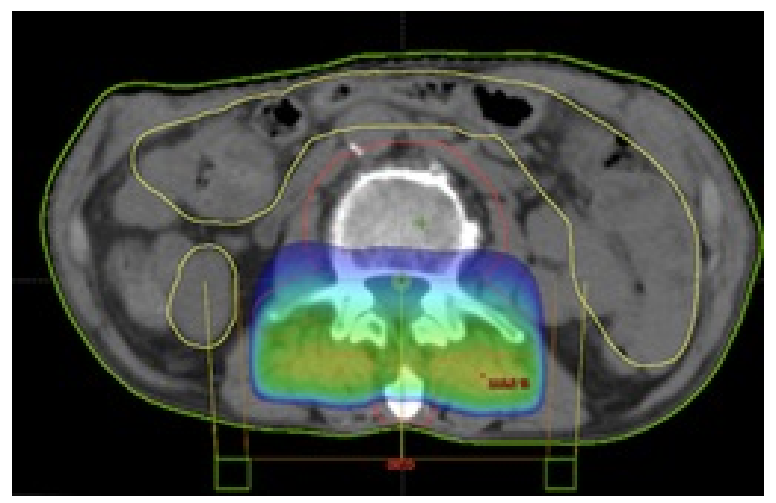

Fig-1: Beam arrangements of one field plan with a $95 \%$ dose color wash. 


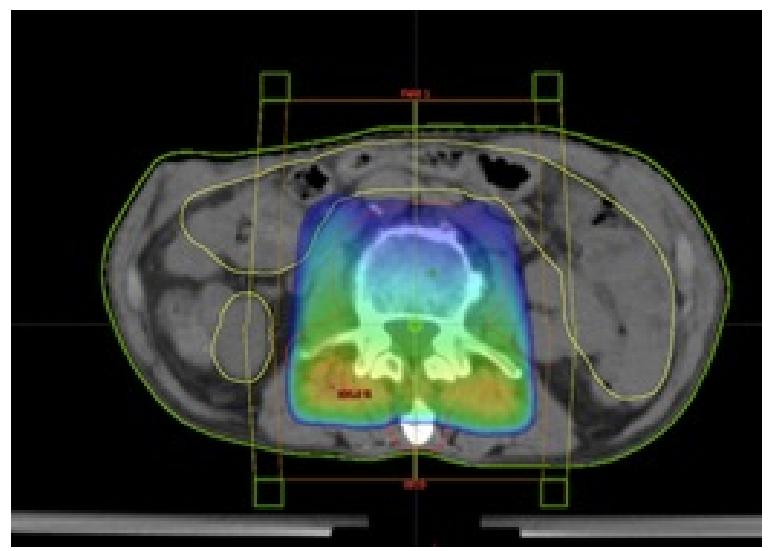

Fig-2: Beam arrangements of two field plans with a $95 \%$ dose color wash.

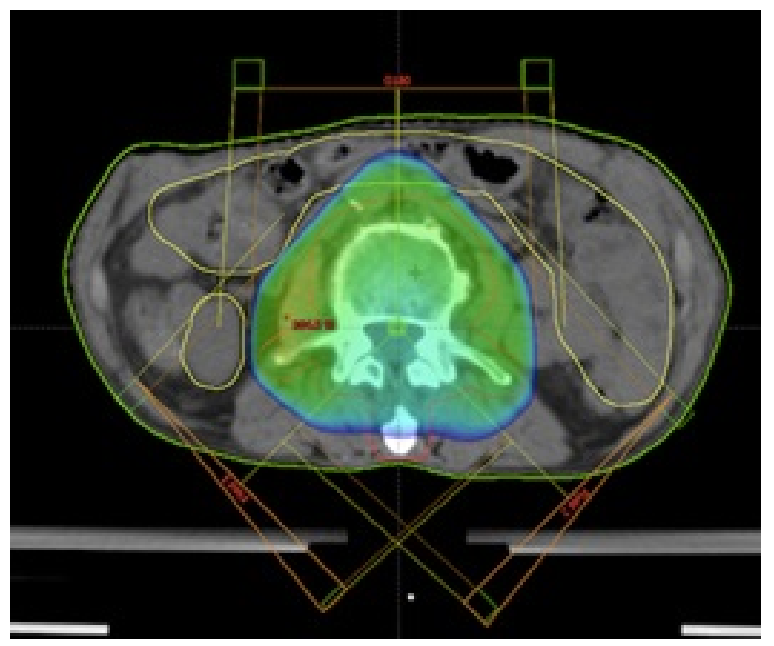

Fig-3: Beam arrangements of three field plan with $95 \%$ dose color wash.

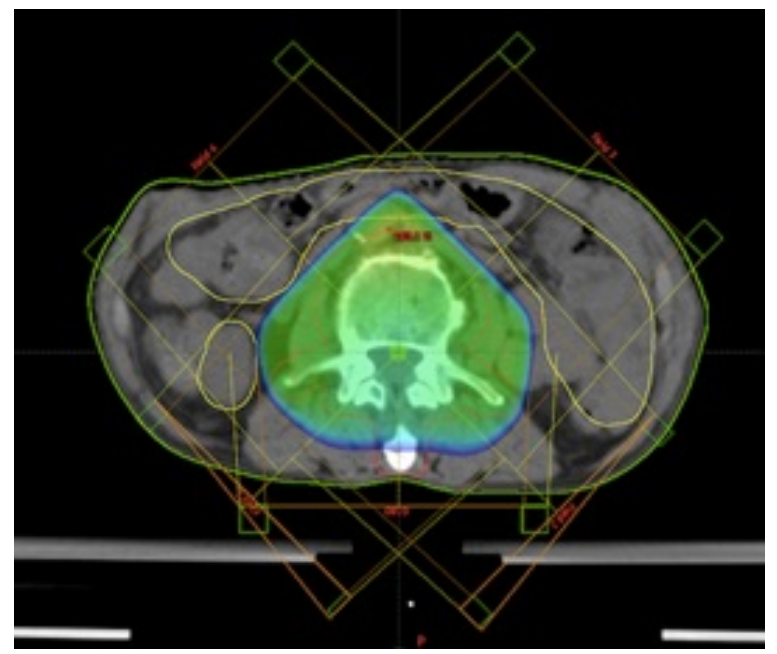

Fig-4: Beam arrangements of three field plan with $95 \%$ dose color wash.

\section{Discussion}

In the present study using various radiotherapy plans the three-field technique showed significantly better coverage of the target volume but with an increase in the dose to organs at risk. reveals that of the four different conformal plans, the three-field techniquewith equally weighing beams gave a significantly better coverage of the target volume of interest. This was achieved at the expense of increasing dose to OAR's.

In terms of dose to OAR's, the maximum rise was observed in dose to kidneys with three field plans compared to one field or two field plans. This is because of the rise in entry dose through partial volumes of kidneys because of posterior oblique beam arrangements. The QUANTEC (Quantitative Analysis of Normal Tissue Effects in Clinic) recommends meaning dose constraints of 15 to 18 Gy to kidneys at 2 Gy per fraction [7].

Considering an alpha /beta ratio of 3 for late radiation-induced nephropathy, the mean dose of 8.72 Gy @ 3Gy/fraction translates into a BED of $11.24 \mathrm{~Gy}$. This increased dose to kidneys is well within tolerance limits. Hence forth, this increment in dose to kidneys does not warrant serious concern as it is unlikely to translate to radiation-induced nephropathy.

The dosimetry of bowel showed a significant rise with two field techniquesover one field technique but the difference was very small in terms of absolute dose with an average increment of only $1.59 \mathrm{~Gy}$. In three field plans, the mean dose was nearly comparable to that of two field plans but was significantly less to that of five field techniques.

The possible explanation of rising with five field techniquesbeing an inevitable rise in entry and exit dose with paired anterior oblique and posterior oblique beams. The fact needs to be acknowledged that QUANTEC recommends restricting the dose of bowel receiving $45 \mathrm{~Gy}$ to be restricted to $195 \mathrm{cc}$ when entire space in the peritoneal cavity is evaluated while prescription was upto 30 Gyonly.

Hence forth, the significant rise with multifield approaches over one field approach does not warrant any serious concern due to less likelihood of clinical manifestations. The mean dose to the esophagus with three field techniquesin 3 Gy equivalent dose was 18.33 Gy. Considering an alpha/beta ratio of 10 for acute complications and 3 for chronic complications, the dose to esophagus 
Translates to a BEDof 18.65 Gy for acute complications and 19.04Gy for chronic complications. These values are less than QUANTEC recommended a mean dose of 34 Gy (EQD2) forthe esophagus.

Similar considerations apply to lungs as the mean dose to lungs was 9.40 Gy that translates to a BED of $12.31 \mathrm{~Gy}$ (alpha/beta $=3$ ) which is below the recommended dose constraint of 13 Gy (EQD2) for lungs. The present study was underpowered to detect any statistically significant difference in the dosimetry of lungs and esophagus between the four techniques as only three patients had thoracic vertebral metastasis however the data shows dose to these OAR's was within tolerable limits with all the plans.

The use of five field plans did not offer any additional advantage over the three fields plans in terms of coverage of the PTV. However, there was a huge inevitable rise in the entry dose and exit dose because of multibeam arrangement reflected in a significantly higher dose of kidneys and bowel.

In the present study, even the two field plans proved to be significantly better over one field plan in terms of coverage of PTV in terms of the significant rise in D90, Dmean, and D50 but were accompanied by a rise in dose to OAR's. A study by Andic $F$ et al. showed similar results with better dose distribution within the intended dose range as per the ICRU 50 recommendations and more homogeneity with two field approach over one field approach.

Their study also showed a significant rise in mean dose to bowels and intestines but the rise was considered to be within reasonable limits in accordance with our interpretation [8]. A three-arm study done by Yeo SGcompared a single PA field, opposed anteroposterior (AP)/PA fields, and the third one with one posteroanterior (PA) field and two posterior oblique fields.

Their study also proved the single PA field to be inferior to AP/PA fields in terms of accomplishing homogeneous target dose distribution and AP/PA fields approach proved to be inferior to the threefield approach that validates the findings of the present study supporting three-field approach. In their study, the lung dose was negatively impacted to a minimal extent but better sparing of the esophagus was seen [9].
Bone metastasis using three-beam plans reveals optimistic results. The limitations of the present study are lack of clinical correlation and smaller sample size.

Therefore, before the clinical adoption of the threefield approach, its efficacy in terms of patientreported outcomes (pain relief, recovery of function) needs to be tested in randomized settings over the standard one field and two field approaches.

\section{Conclusion}

The use of a higher number of fields that are two fieldsand three fields approach ensures significantly better coverage over the usually practiced one field approach in palliative radiotherapy of bony metastasis. The increase in dose to surrounding OAR's with multibeam approaches was within acceptable limits. This may translate into better clinical outcomes with palliative radiotherapy along with an acceptable side effect profile. Its correlation with clinical outcomes demands further research in clinical settings. Contrarily, the use of five field approach adds to treatment complexity with no significant advantage in terms of dosimetry over the three field plans.

\section{What does the present study add to existing knowledge}

Increasing the number of fields in the 3DCRT technique has the potential to provide optimal coverage to the target region with nearly $97 \%$ of the dose received by $95 \%$ of the target volume. The increased dose to OAR's with the multibeam arrangement was well within tolerance limits.

\section{Author's contribution}

Dr. Naina Gupta: Collected data, manuscript
drafting

Dr. Pavan Kumar: Verification of data, Manuscript drafting, and editing

Dr. Piyush Kumar: Study methodology, manuscript drafting, and editing

Mrs. Navitha S: Treatment planning

Dr. Ankita Mehta: Manuscript drafting, data analysis

Dr. Arvind Chauhan: Manuscript drafting

The utilization of 3DCRT techniques in palliation of 


\section{Reference}

01. Chin H, Kim J. Bone Metastasis- Concise Overview. Federal Practitioner. 2015;32(2)24. [Crossref]

02. Cleeland C, von Moos R, Walker MS, Wang $Y$, Gao J, Chavez-MacGregor $M$, et al. Burden of symptoms associated with development of metastatic bone disease in patients with breast cancer. Support Care Cancer. 2016;24(8)35573565.

doi: Epub 2016 Mar 29 [Article] [Crossref]

03. Kumar A, Mukundan $H$, Bhatnagar S, Sarin A, Taneja S, Sahoo S. Radiation for Palliation- Role of Palliative Radiotherapy in Allevieating Pain/Symptoms in a Prospective Observational Study at Two Tertiary Care Centers. Indian J Palliat Care. 2019;25(3)391-397. doi: [Article] [Crossref]

04. Macedo F, Ladeira K, Pinho F, Saraiva N, Bonito N. , Pinto $L$, et al. Bone Metastases- An Overview. Oncol Rev.

2017;11(1)321. doi: [Article] [Crossref]

05. Cheng YC, Ueno NT. Improvement of survival and prospect of cure in patients with metastatic breast cancer. Breast Cancer. 2012;19(3)191199.

doi: [Article] [Crossref]

06. International commission on radiation units and measurements. Prescribing, recording, and reporting photon beam therapy, $\mathrm{P} \mathrm{Cm}$ - (ICRU report; 62) "Supplement to ICRU Report 50. " Journal of the ICRU. (1999).

[Crossref]

07. Radiation Oncology/Toxicity/QUANTEC. Wikibooks, The Free Textbook Project. QUANTEC. (2015, September 23) 18:14 UTC Page Version ID: 2996027.

[Crossref]

08. Andic F, Baz Cifci S, Ors Y, Niang U, Dirier A, Adli M. A dosimetric comparison of different treatment plans of palliative spinal bone irradiation- analysis of dose coverage with respect to ICRU 50 Report. J Exp Clin Cancer Res. $2009 ; 28(1) 2$.

doi: [Article] [Crossref]
09. Yeo SG. Palliative radiotherapy for thoracic spine metastases- Dosimetric advantage of threedimensional conformal plans. Oncol Lett. 2015;10(1)497-501.

doi: [Article] [Crossref] 